\title{
SIMULATION OF A PERFECTLY DIELECTRIC DROP IN ELECTRO-OSMOTIC FLOW OF AN ELECTROLYTE THROUGH A MICROCHANNEL
}

\author{
M. M. B. FELISBERTO ${ }^{1}$ and A. V. N. C. TEIXEIRA ${ }^{2}$ \\ ${ }^{1}$ Universidade Federal de Viçosa, Departamento de Química \\ ${ }^{2}$ Universidade Federal de Viçosa, Departamento de Física \\ E-mail: michelle.felisberto@ufv.br
}

\begin{abstract}
This research uses a computational fluid dynamic model to simulate motion and deformation of a dielectric drop in electrolyte solution in a microchannel. Different values of wall charge density, the Debye-Hückel parameter and the Weber number (We) were used for uncharged, positively and negatively charged drop interfaces. Drop flow and deformation were analysed and the effects of charge distribution, electric field and permittivity jump were discussed. For a positively charged channel wall, negatively charged drops moved faster and positively charged drops moved slower than an uncharged drop. This effect increased for a higher We. Vortex flows were observed inside the drop. For a low surface tension, the drops were elongated due to electric forces acting on its surface with the charged drops deforming more than the uncharged one. When the permittivity component of the force was removed, the drop had a horizontal deformation that was sufficiently high to cause the negatively charged drop to break up.
\end{abstract}

KEYWORDS: Computational fluid dynamic (CFD); Dielectric drop; Electro-osmotic flow; Electrolyte solution; Microchannel.

\section{INTRODUCTION}

Many areas, such as biochemical and chemical analysis and emulsions, study the fundaments of electro-osmotic flow and electrical properties in microchannels. These studies lead to applications as soil remediation (Cameselle, 2015), lab-on-a-chip development ( $\mathrm{Li}$, 2004) and electro-osmotic pumps (Wang et al., 2009). The prediction of the behavior of droplets in microfluidic and nanofluidic applications is an important part of these studies, especially regarding the colloid industry (Huang and Lee, 2012).

Various numerical models have been developed to describe these microsystems accounting for electro-osmotic flow, electrical forces and liquid-liquid interfaces. Berry and co-workers (Berry et al., 2013) presented a numerical model for electrokinetic flow of multiphase systems with deformable interfaces based on a combination of the level set and volume of fluid techniques. The presence of electrolyte solutions may cause the drop interface to carry charge. Davidson and co-workers (Davidson et al., 2014) used an adapted method for capturing interface boundary conditions proposed by Liu and co-workers (Liu et 
al., 2000) to extend the previous numerical model to account for the effect of interfacial charges. This extension allows the analysis of the electrokinetic forces effect on the deformation of a charged drop in an electrolyte solution.

This research project studied the special case of a cylindrical microchannel with positively charged walls where a perfectly dielectric drop with a charged surface is placed inside an electrolyte. The solution and drop flow is driven by an applied electric field. When either the channel walls or the drop interface are charged, electrokinetic phenomena are developed in the microchannel. These phenomena generate charge redistribution due to advection, conduction of ions and diffusion and therefore affect the drop deformation in the channel. Viscous and electrical forces act on the drop accounting for its behavior regarding the motion inside the channel and the deformation. The model was predicted using a computational fluid dynamic (CFD) numerical model (Berry et al., 2013) that was modified to account for charge density at the drop and liquid interface (Davidson et al., 2014).

Firstly, a literature review will be presented, containing the history and applications of the electro-osmotic flow and the behaviour of liquid droplets inside microchannel. Subsequently, a theoretical background will be given with all the mechanical and electrical concepts applied in this study as well as the governing equations, dimensionless groups and an explanation of the numerical model. Then the conditions of the case study will be set up and the results of the numerical simulation will be shown and discussed. Lastly, the study will be concluded and suggestions for further analysis will be made.

\subsection{Literature review}

\subsubsection{Electro-osmotic flow history and applications}

The electrokinetic phenomenon was first observed by Reuss in 1809, when he observed that water moved through soil pores when an electric potential was applied (Li et al., 2013, Pillai et al., 2015, Wang et al., 2009). In the mid and late 1900 this effect was more widely studied and applied in fields such as drug delivery, chemical analysis (Wang et al., 2009) and soil stabilization (Pillai et al., 2015). Over the past years, these phenomena have been widely studied by researchers and applied in many engineering and analytical fields. A variety of numerical methods have been developed to predict electrokinetic and electrohydrodynamic problems. These models are usually based on combinations of the Navier-Stokes equation for fluid flow and the Poisson-Boltzmann equation for electric potential distribution (Li et al., 2013). Amongst the applications of electro-osmotic flow are: electrokinetic treatment of contaminated soil (Cameselle, 2015, Sahoo et al. 2009), electro-osmotic pumps (Li et al., 2013, Wang et al., 2009), flow mixing in microchannel (Peng and Li, 2015) and electric field-driven microreactor (Susarrey-Arce et al., 2015).

The removal of heavy metal contaminants from soils through conventional technologies is extremely hard and the electrokinetic models come as good alternative to this procedure. Experimental (Cameselle, 2015, Sahoo et al., 2009) as well as numerical models (Gomes et al., 2015) have been developed to describe the efficiency of transportation of ions in soil pores using electric field-driven flows. It was found that chemical nature of soil particles, ionic concentration and ionic strength of inertial fluids, $\mathrm{pH}$, temperature and electrochemical 
reactions can influence the development and maintenance of electro-osmotic flow (Cameselle, 2015).

Electro-osmotic pumping have been found to be more effective and advantageous on micro-porous systems than pressure-driven mechanisms alone (Li et al., 2013). A few of the primary advantages of this application are the absence of mechanical moving parts of the pump, the possibility to instantly switch flow directions and the capability of generating constant pulse free flows (Wang et al., 2009).

An active alternative to enhance fluid mixing efficiency is to introduce an external energy source to the mixer and one way of doing it is by applying an electric field to generate electro-osmotic flow (Peng and Li, 2015). In addition to increasing the flow, this method can also generate vortexes and generate turbulence for a chaotic mixing. (Peng and Li, 2015) studied the influence of ionic concentration on electro-osmotic flow mixing and the dependence of zeta potential, dielectric constant and electric conductivity from this parameter. It was found that, for sufficiently high ionic concentrations (higher than $1 \mathrm{M}$ for their case study), extremely low electro-osmotic flow mobility and non-uniform electric field and velocity flows were observed. Similar results were also found on this research project.

Regarding microreactor, electric field controlled surfaces can have an effect on adsorption and desorption processes relevant to heterogeneous catalysts, when these are in contact with electrolyte solutions (Susarrey-Arce et al., 2015).

Studies have found that pressure-driven flows through microchannels are influenced by electrokinetic effects (Davidson and Harvie, 2007). When pressure-driven fluids contain ions and/or microchannels are electrically charged, hydrodynamic resistance can increase due to a flow-induced electric field resulting from streaming potential, the so-called electroviscous effect (Davidson and Harvie, 2007).

\subsubsection{Liquid droplets inside microchannels}

The manipulation of liquid droplets inside microchannels is an important part of the electrokinetic phenomena. It is present in colloid and emulsion industries, especially in nanofluidic and microfluidic. Some potential applications are microcapsules, microreactors, micromixers, lab-on-a-chip and emulsion electrokinetic chromatography (EKC) (Huang and Lee, 2012). The movement of a charged particle relative to a surrounding fluid in response to an applied electric field is called electrophoresis. The principles and equations that describe this phenomena are the same as the electro-osmotic flow ( $\mathrm{Li}, 2004)$ and this method is believed to have a great potential in the manipulation of droplets (Huang and Lee, 2012).

Lac and Sherwood (Lac and Sherwood, 2009) predicted the influence of a suspended drop in the generation of streaming potential in capillary pressure-driven flow. It was found that the presence of a non-conduction drop can increase the streaming potential by decreasing the average conductivity of the capillary but it can also decrease the streaming potential when it has a lower viscosity than the surrounding fluid, which reduces the pressure difference and 
hence the streaming current. In their study, the drop surface charge was neglected, which makes it inappropriate for the case of a conducting drop.

Furthermore, the motion of a liquid droplet in a cylindrical pore was studied by Huang and Lee (Huang and Lee, 2012) with an arbitrary surface potential and electric double layer thickness. A vortex flow was observed due to electric driving force and it was found that a charged wall with opposite sign enhanced drop mobility while same signal charge deterred it. Similar results were also found in this research project, however, different boundary conditions led to divergent conclusions regarding the effect of the electric double layer (EDL) thickness on drop mobility. While Huang and Lee (Huang and Lee, 2012) defended that thicker EDL lead to a weaker electro-osmotic flow for having lesser ions per unit volume, their research found that a thicker electric double layer actually enhanced drop mobility. This can be due to the fact that despite having fewer ions, a thicker electric double layer actually has a greater contact area with the drop and therefore generates more electro-osmotic flow. In addition, Peng and Li (Peng and Li, 2015) studied the effect of ionic concentration on electro-osmotic flow mixing and discovered that higher concentration of ions led to lower electro-osmotic flow, which is consistent with the results presented in this report.

When one of the fluids inside the microchannel (drop fluid or continuous media) is an electrolyte, the drop can also have a deformable surface. This happens because electric forces act on the surface of the drop in opposition to the surface tension. These forces are caused by charge distribution in the electrolyte solution or permittivity jump across the interface, when fluids with different permittivity are contacted. They are also influenced by the presence of electric field in the microchannel. Pillai and co-workers (Pillai et al., 2015) investigated the deformation and breakup of conducting drops in non-conducting media subjected to an external electric field. The presence of free charges inside the drop created opposing conductive and diffusive fluxes for cations and anions which led to complex ion behaviour inside the drop causing its deformation and eventual breakup. This research project investigate the case of a non-conduction drop in a conducting media, which could be the case of an oil drop in water and less drastic drop deformation was observed when compared to the work of Pillai and co-workers (Pillai et al., 2015).

The presence of an electrolyte solution in the microchannel also causes the liquid/liquid interface to carry electric charges due to the adsorption of ions to the surface. The electrokinetic flow model for multiphase flow extended to account for interfacial charges presented by Berry and co-workers (Berry et al., 2013) and Davidson and co-workers (Davidson et al., 2014) allows for a better prediction of drop mobility and deformation as well as a comprehensive study of the electrokinetic forces acting on the surface of the drop. This model has recently been validated using analytical solutions and experimental results from published literature (Davidson et al., 2015) and were shown to have accurate predictions. The advances presented by the authors in electrokinetic numerical modelling of microfluidic systems represent an important step towards understanding liquid droplet behaviours in microchannels, which is crucial to successful design and operation of numerous applications. 


\subsection{Theoretical background}

\subsubsection{Electrical double layer and Electro-osmotic flow}

The first concept that needs to be introduced in order to understand the electrokinetic phenomena inside the microchannel is the electrical double layer. When a solid or liquid surface is in contact with an aqueous solution, its electrical static charges attract counter ions in the solution to the region close to the surface to balance the charges (Li, 2004). This region, where there is an accumulation of counter ions is called electric double layer (EDL). This double layer is composed of two layers. The compact layer, where the ions are immobile and strongly attached to the surface is the layer closest to the surface, called Stern layer. The second layer, called diffuse layer, is where the ions are mobile and the net charge gradually reduces to zero, as the distance from the surface gets larger. Zeta potential, $\zeta$, is the electrical potential difference at the separation point of the Stern and diffuse layer and it represents a measure of the electrical charges of particles suspended in liquid. It is considered a constant approximation of the surface electric potential in electrokinetic models (Li, 2004) .

The thickness of the EDL is a function of the bulk ionic concentration and electrical properties of the liquid and is independent of the solid surface properties ( $\mathrm{Li}, 2004)$. As seen on the dimensionless parameter inverse Debye length shown by Equation 1:

$$
\kappa^{-1}=\left[\frac{\varepsilon_{0} \varepsilon_{\mathrm{ref}} k T}{2 z^{2} e^{2} n_{0} R^{2}}\right]^{1 / 2}
$$

where $\varepsilon_{0}$ and $\varepsilon_{\text {ref }}$ are the permittivity in vacuum and in the material, respectively, $k$ is the Boltzmann's constant, $T$ the temperature, $z$ is the number of charges, $e$ is the elementary charge, $R$ is a characteristic length, the actual thickness of the EDL is inversely proportional to the bulk ionic concentration, $n_{0}$. This means that for higher ion concentration, the ions are strongly attracted to the region close to the charged surface, the EDL gets more "compressed" and therefore is thinner.

When an electric field is applied tangentially to the EDL an electric body force is exerted on the counter ions of the diffuse layer of the EDL. The counter ions start to move pulling the liquid with them. The rest of the liquid moves through the channel by viscous forces. This is known as electro-osmotic flow (Li, 2004). As seen in Figure 1, the velocity profile of a fully developed electro-osmotic flow is very peculiar and different to the parabolic profile found on viscous flow. In this case, the velocity is zero on the surface, it increases within the EDL, reaches a maximum and becomes a constant in the bulk solution. 


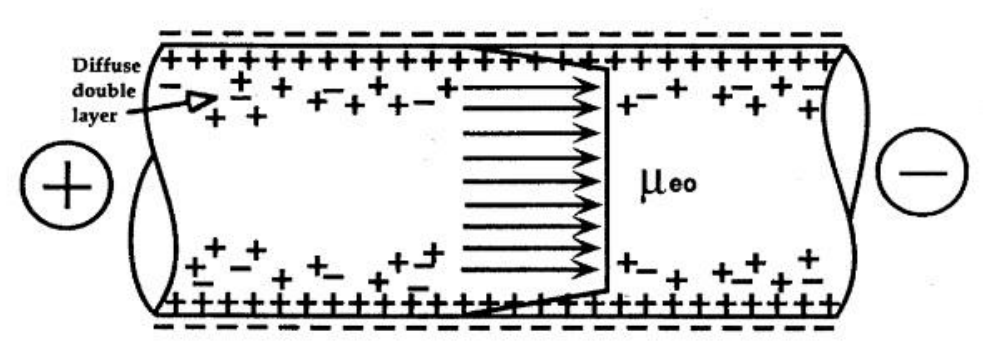

Figure 1. Velocity profile of a fully developed electro-osmotic flow.

\subsubsection{Governing equations and dimensionless groups}

The LS-VOF-based numerical method uses the following equations to describe fluid behaviour in the microchannel (Davidson et al., 2015):

$$
\begin{aligned}
& \frac{\partial \phi}{\partial t}+\nabla \cdot(\boldsymbol{v} \phi)=0 \\
& \boldsymbol{\nabla} \boldsymbol{v}=0 \\
& \frac{\partial \rho \boldsymbol{v}}{\partial t}+\boldsymbol{\nabla} \cdot(\rho \boldsymbol{v} \boldsymbol{v})=-\boldsymbol{\nabla} P+\frac{1}{R e} \boldsymbol{\nabla} \cdot \boldsymbol{\tau}+\frac{1}{W e} \delta s \boldsymbol{f}_{s}+\frac{C a_{E}}{W e} \boldsymbol{\nabla} \cdot \boldsymbol{\tau}_{\boldsymbol{m}} \\
& \boldsymbol{\tau}=\mu\left[\boldsymbol{\nabla} \boldsymbol{v}+(\boldsymbol{\nabla} \boldsymbol{v})^{\mathrm{T}}\right] \\
& \boldsymbol{\tau}_{\boldsymbol{m}}=\varepsilon \boldsymbol{E} \boldsymbol{E}-\frac{1}{2} \varepsilon(\boldsymbol{E} \cdot \boldsymbol{E}) \boldsymbol{I}
\end{aligned}
$$

Equations 2 and 3 describe the fluid flow in terms of the velocity $\boldsymbol{v}$ and the discrete phase volume function $\phi$. Equation 4 is the momentum equation, where the velocity is in terms of pressure $P$, the viscous stress tensor $\tau$ (Equation 5), where $\mu$ is the fluid viscosity, and two additional terms that account for surface force due to interfacial tension $\left(f_{s}=\right.$ surface force and $\delta s=$ delta function for interfacial tension) and electric force via Maxwell stress tensor $\boldsymbol{\tau}_{\boldsymbol{m}}$ (Equation 6). $\rho$ and $\varepsilon$ are fluid density and permittivity, respectively. Gravitational terms are omitted for being irrelevant for the momentum in microscale.

$$
E=-\nabla U
$$




$$
\boldsymbol{\nabla} \varepsilon . \boldsymbol{E}=\frac{1}{2} \kappa^{2}\left(n_{+}-n_{-}\right)+S_{q}
$$

Equation 7 is the usual relation of the electric field $\boldsymbol{E}$ and the electric potential $U$. Equation 8 is the Poisson equation and it brings on its right side the dimensionless charge per unit volume due to free ions $\rho_{e}=n_{+}-n_{-}$and the charge per unit volume due to interface charge density, $S_{q}$. In the numerical formulation the subscript $q$ is replaced to $w$ or $i$ to specify the wall or drop surface respectively.

The ion transport is described by Equation 9, which incorporates advection, diffusion and conduction of ions. $n_{+}$and $n_{\text {- }}$ are the cations and anions concentrations in the electrolyte solution, respectively (Davidson et al., 2015).

$$
\frac{\partial \phi n_{ \pm}}{\partial t}+\nabla \cdot\left[\phi n_{ \pm}\left(v \pm \frac{1}{\mathrm{Pe}} \boldsymbol{E}\right)\right]=\frac{1}{\mathrm{Pe}} \boldsymbol{\nabla} \cdot\left(\phi \boldsymbol{\nabla} n_{ \pm}\right) .
$$

To account for interfacial charges, two methods were evaluated elsewhere (Davidson et al., 2014), which are the delta function formulation and another one adapted from (Liu et al., 2000) that accounts for jump in electric displacement due to interfacial charges and discontinuity in permittivity across interface. Davidson and co-workers (Davidson et al., 2014) concluded that the second one was more satisfactory to represent the electrical potential jump in the interface. Explicit expressions and formulations for the method can be found in (Davidson et al., 2014, Liu et al., 2000).

All the physical variables are nondimensionalized using reference values, giving dimensionless channel and drop radius $R$ and $r$, respectively, electric field $E$, permittivity $\varepsilon$, surface tension $\gamma$, density $\rho$ and viscosity $\mu$. The time scale $t$, the velocity $v$ and the free ions

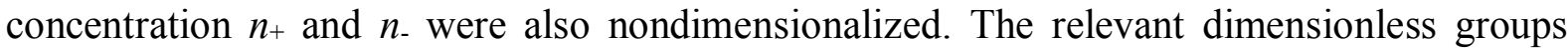
seen on the governing equations are as follows, together with Equation 1 (the inverse Debye length):

$$
\mathrm{Re}=\frac{\rho_{\mathrm{ref}} v R}{\mu_{\mathrm{ref}}} \quad \mathrm{We}=\frac{\rho_{\mathrm{ref}} v^{2} R}{\gamma_{\mathrm{ref}}} \quad \mathrm{Ca}_{\mathrm{E}}=\frac{\varepsilon_{0} \varepsilon_{\mathrm{ref}} E_{\mathrm{ref}}^{2} R}{\gamma} \quad \mathrm{Pe}=\frac{v R}{D_{\mathrm{ref}}}
$$

where Reynolds number ( $R e)$ is the ratio between inertial and viscous forces indicating laminar or turbulent flow; Weber number (We) is the ratio of the inertial forces and the surface tension forces; the electric capillary number $\left(\mathrm{Ca}_{\mathrm{E}}\right)$ is the ratio of viscous forces and 
the interface tension forces; the Péclet number $(\mathrm{Pe})$ is defined as the ratio of the advection rate to the diffusion rate of a physical quantity. The inverse Debye length represents the distance of the electrostatic effect of a charge carrier in a solution. $\varepsilon_{0}, D, e, z, k$ and $T$ are respectively the permittivity of free space, diffusivity, the elementary charge, the valence, Boltzmann constant and absolute temperature.

\subsubsection{Numerical formulation}

The numerical model presented by Berry and co-workers (Berry et al., 2013) is used for electrokinetic flow of multiphase systems with deformable surfaces. Described in details by Rudman (Rudman 1998), this method is based on a level set-volume of fluid algorithm and features a multiphase formulation of the ion transport equation that accounts for advection, diffusion and conduction of charge carriers within each phase. The electric force present in the momentum equation is accounted for by the Maxwell electric force. A modification on the Nernst-Planck equation for ion transport was made to implement an ion-impenetrable boundary condition and ensure that there would be no ion flux across the interface. The calculation is performed in a two-dimensional axisymmetric staggered grid and the algorithm is an explicit time stepping method with time steps defined dynamically to satisfy stability limits (Davidson et al., 2015).

The presence of charged interfaces causes an associated jump in the electric displacement, which was not accounted for in the initial numerical method. Recently, Davidson and coworkers (Davidson et al., 2014) extended the method to account for surface charge. The new algorithm was based on a method developed by Liu and co-workers (Liu et al., 2000) that incorporates jump conditions across surfaces. The electric field is solved in the Poisson equation (Equation 8) with a representation of the surface charge $\mathrm{S}_{\mathrm{q}}$. Detailed expressions for the method can be seen in (Davidson et al., 2014), (Liu et al., 2000) and (Davidson et al., 2015).

Previous works using this numerical method have shown that a grid resolution of 32 cells is necessary to accurately predict the drop shape (Pillai et al., 2015), therefore this resolution was used in this work. However, a grid refinement showed that a grid resolution of 64 cells could cause a variation of drop velocity of approximately $10 \%$, showing that this could be a potential limitation to the prediction of drop behaviour.

\section{METHODOLOGY}

\subsection{Initial conditions}

In this project, specific cases were considered with constant dimensionless parameters. A cylindrical microchannel, whose length scale was defined as the channel radius. In that way the total length was set as $=14$. The cannel was considered to be filled with an electrolyte solution and a perfectly dielectric drop of radius 0.5 was placed in its center. The flow was driven by a downwards electric field adjusted to Reynolds $=0.01$. The reference electric field,

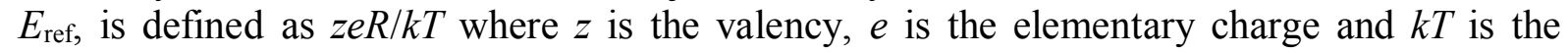
thermal energy. The electric field in the experiment is set to be $E=10$, meaning that the electric field is 10 times the reference electric field $E_{\text {ref. }}$ The permittivity ratio between the 
drop and the continuous solution was $\varepsilon_{d} / \varepsilon_{\mathrm{c}}=0.025$. The dimensionless density and viscosity of the drop and continuous solution were adjusted to $\rho_{d}=\rho_{c}=1$ and $\mu_{d}=\mu_{c}=1$, which is equivalent to redefining the mass and time scales, respectively.

Debye-Hückel parameters of $\kappa=2$ and $\kappa=8$ were considered. For each $\kappa$ the surface wall was charged with increasing charge density: $S_{w}=0,4,8$ and 16. For each of these cases, the drop interface was either positively or negatively charged, with fixed surface charge density $S_{i}=+8$ and $S_{i}=-8$, respectively, or uncharged, $S_{i}=0$. This configuration is shown in Figure 2 .

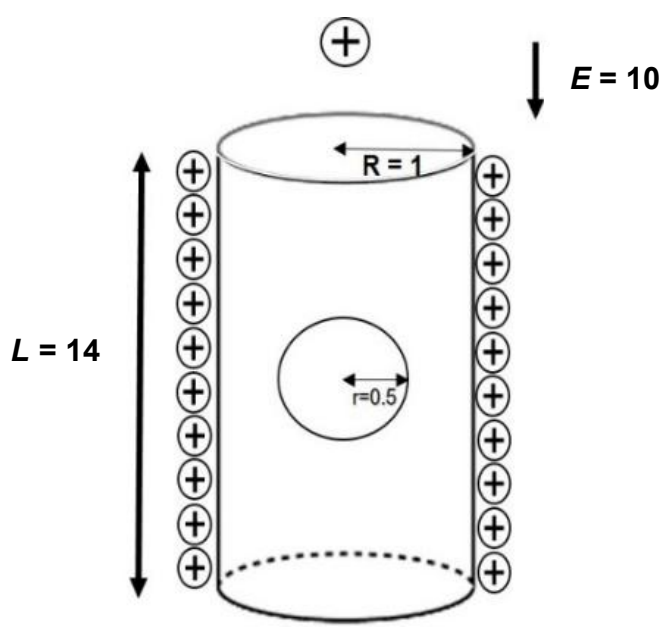

Figure 2. Problem set up: cylindrical microchannel of radius 1 , length 14 and positively charged walls. Flow driven by a downward electric field of magnitude 10 and a dielectric drop of radius 0.5 on the central axis.

\subsubsection{Case study one: Perfectly spherical drop}

In order to study the influence of wall and drop surface charge density and the thickness of the EDL on the motion of the drop, the Weber number was set to We $=0.001$, sufficiently low to give a perfectly spherical drop at steady state due to the high surface tension associated with a low Weber number. At steady state, the electro-osmotic flow of the solution, drop velocity, electric field and charge distribution were analysed.

\subsubsection{Case study two: Drop with deformation}

In the second case study, the surface tension of the drop was decreased to account for drop deformation. While all the other parameters were kept constant and equal to the case study one, the Weber number was increased to $\mathrm{We}=0.008$, as this parameter is inversely proportional to the surface tension. The steady state drop deformation, velocity, electric field and charge distribution were analysed. 


\subsubsection{Deformation with no permittivity gradient}

One of the factors that contribute to the deformation of the drop is the permittivity jump between the drop and the continuous solution, which causes a permittivity force to act on the drop surface. To investigate the relevance of this parameter to drop deformation, the cases of We $=0.008, \kappa=8, S_{w}=8$ and $S_{i}=-8,0$ and +8 were investigated with no permittivity jump, or $\varepsilon_{d} / \varepsilon_{c}=1$. The charge distributions in a dynamic state were investigated.

The summary of all the 48 simulated case studies detailed on the topics 2.1.1 and 2.2.2 are presented on Table 1. As described on the topic 2.1.3, the investigation of the permittivity jump influence was performed on three of the cases, which are indicated in red on Table 1.

Table 1. Summary of simulations performed for Case study 1 , Case study 2. *: permittivity jump investigation.

\begin{tabular}{|c|c|c|c|c|c|c|c|c|c|c|c|}
\hline \multicolumn{6}{|c|}{ Case study $1: \mathrm{We}=0.001$} & \multicolumn{6}{|c|}{ Case study $2: \mathrm{We}=0.008$} \\
\hline \multicolumn{3}{|c|}{$\kappa=2$} & \multicolumn{3}{|c|}{$\kappa=8$} & \multicolumn{3}{|c|}{$\kappa=2$} & \multicolumn{3}{|c|}{$\kappa=8$} \\
\hline$S_{i}=-8$ & $S_{i}=0$ & $S_{i}=+8$ & $S_{i}=-8$ & $S_{i}=0$ & $S_{i}=+8$ & $S_{i}=-8$ & $S_{i}=0$ & $S_{i}=+8$ & $S_{i}=-8$ & $S_{i}=0$ & $S_{i}=+8$ \\
\hline \multicolumn{3}{|c|}{$S_{w}=0,4,8$ and 16} & \multicolumn{3}{|c|}{$S_{w}=0,4,8$ and 16} & \multicolumn{3}{|c|}{$S_{w}=0,4,8$ and 16} & \multicolumn{3}{|c|}{$S_{w}=0,4,8^{*}$ and 16} \\
\hline
\end{tabular}

\section{RESULTS AND DISCUSSION}

\subsection{Case study one: Perfectly spherical drop}

\subsubsection{Effect of wall and drop surface charge density on drop velocity}

When the external electric field was applied through the channel, the electrical forces acting on the free ions from the electric double layer caused the fluid to move upwards in an electro-osmotic flow. Consequently, the drop was carried along in the same direction. However, if the surface of the drop was charged, an electric double layer was also formed around the drop and the electrical forces acting due to these free ions either enhanced or decreased the drop movement relative to the fluid. Figure 3 shows the dimensionless velocity of the drop for the proposed cases. The negative velocities for the cases where there was no wall charge and the drop surface was positively charged showed that when there was no electric double layer near the charge driving the fluid upwards, the electrical force surrounding the drop was sufficient to drag it down towards the anode. 


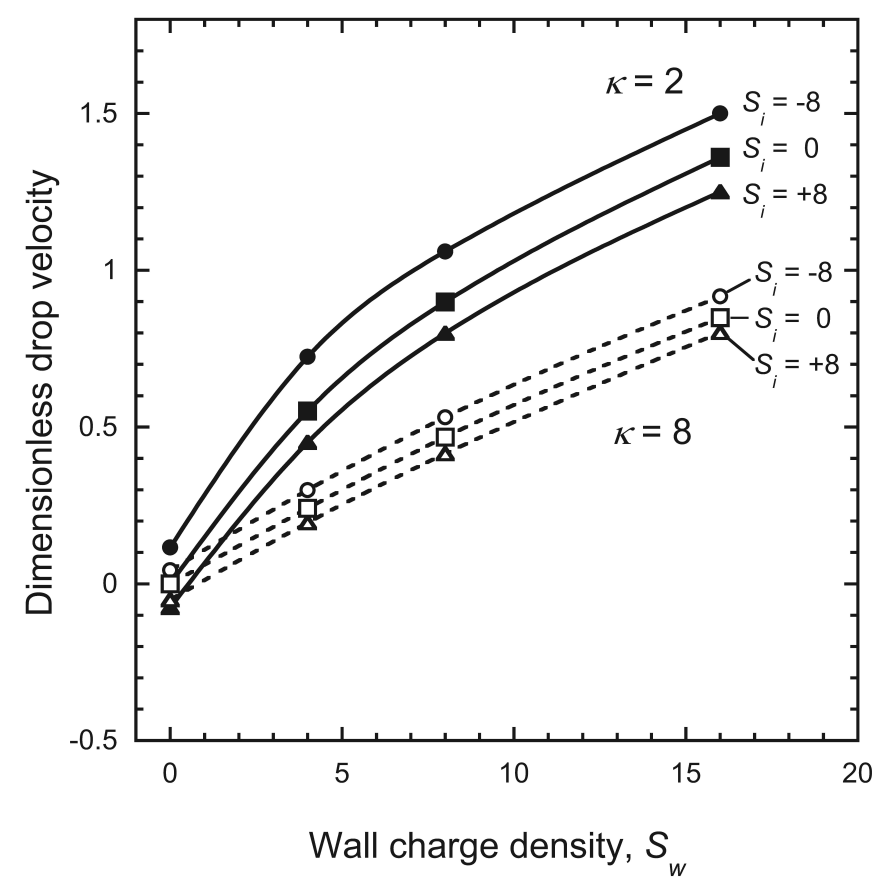

Figure 3. Dimensionless drop velocity at steady state for $\mathrm{We}=0.001$. The lines are guides for the eye. The continuous lines and filled symbols corresponds to $\kappa=2$, and the dashed lines and opened symbols corresponds to $\kappa=8$. In both cases the circles are for $S_{i}=-8$, squares for $S_{i}=0$ and triangles for $S_{i}=+8$.

From the results shown in the Figure 3 a) and b), it was evident that for a higher $\kappa$, in other words a thinner electrical double layer, the magnitude of the velocity of the drop was generally smaller. A possible explanation to this is that for a higher $\kappa$, the ion concentration in the bulk solution is higher which causes the free ions in the diffuse layer of the electrical double layer to be more 'compacted' and closer to the charged surface. In addition to the compaction, the area in which the ions move to create the flow is smaller, therefore a weaker electro-osmotic flow is generated. Since we only tested two values of $\kappa$, it is not possible to conclude that this is always the case. In fact, Huang and Lee (Huang and Lee, 2012) studied a similar case with a spherical drop with an electro-osmotic flow in a microchannel and the drop mobility as a function of $\kappa$ was shown to vary significantly when other parameters were altered. It either increased or decreased depending on the range of $\kappa$ and other factors such as viscosity ratio, drop to channel ratio and the zeta potential. On the other hand, when studying electrolyte fluid mixing in a microchannel (Peng and Li 2015) found that higher ionic concentrations gave lower electro-osmotic flow mobility.

Regardless of the drop interface charge density, the higher the wall charge density, the faster the fluid, and consequently the drop moved. This was due to the fact that for a given ionic concentration in the bulk solution (a given EDL thickness), higher charge density attracted more counter ions to the EDL and therefore a stronger electric force resulted in a higher velocity. 
When compared to the uncharged drop surface of same $\kappa$ and same wall charge density, the negatively charged drop moved faster and the positively charged drop moved slower. Since the electric field was applied downwards and the channel walls were positively charged, the EDL near the wall had an excess of negative ions that caused the fluid to move upwards in the direction of the cathode. The negative charges on the drop surface also tended to move towards the positive end of the channel, and therefore the velocity of the drop was enhanced. Similarly, the positive charge density on the drop surface would tend to move away from the positive end of the channel and therefore the overall drop velocity was decreased. This result was consistent to that presented by Huang and Lee (Huang and Lee, 2012) as it was found that for a given $\kappa$, a positively charged wall slowed a positively charged drop down and a negatively charged wall enhanced its velocity. In conclusion, the drop velocity will be enhanced when drop surface and wall have opposite charges and it will be decreased when they have the same charge.

\subsubsection{Electro-osmotic flow and vortex flow}

The velocity profile of the fluid inside the channel was consistent to what is expected in an electro-osmotic flow, especially for a thinner electric double layer, as shown in Figure 4 a) and b). The velocity was zero on the surface and increased significantly along the diffuse layer of the electric double layer.

For $\kappa=8$, Figure 4 a), it reached a constant velocity in the bulk solution and showed a plug-flow profile. On the other hand, for $\kappa=2$, Figure $4 \mathrm{~b}$ ), the electric double layer was too thick compared to the channel radius and therefore a plug flow is not observed in the bulk solution. The final velocity of the fluid increased proportionally to the wall charge density. Figure 4 shows the velocity profiles of the fluid inside the channel positioned away from the drop for $\kappa=8$ and $\kappa=2$ whilst increasing the wall charge density. These results were similar regardless of charge density of the drop surface as it is independent of this parameter. It is also evident from Figure 4 that a thicker EDL (smaller $\kappa$ ) generated greater electro-osmotic flow.
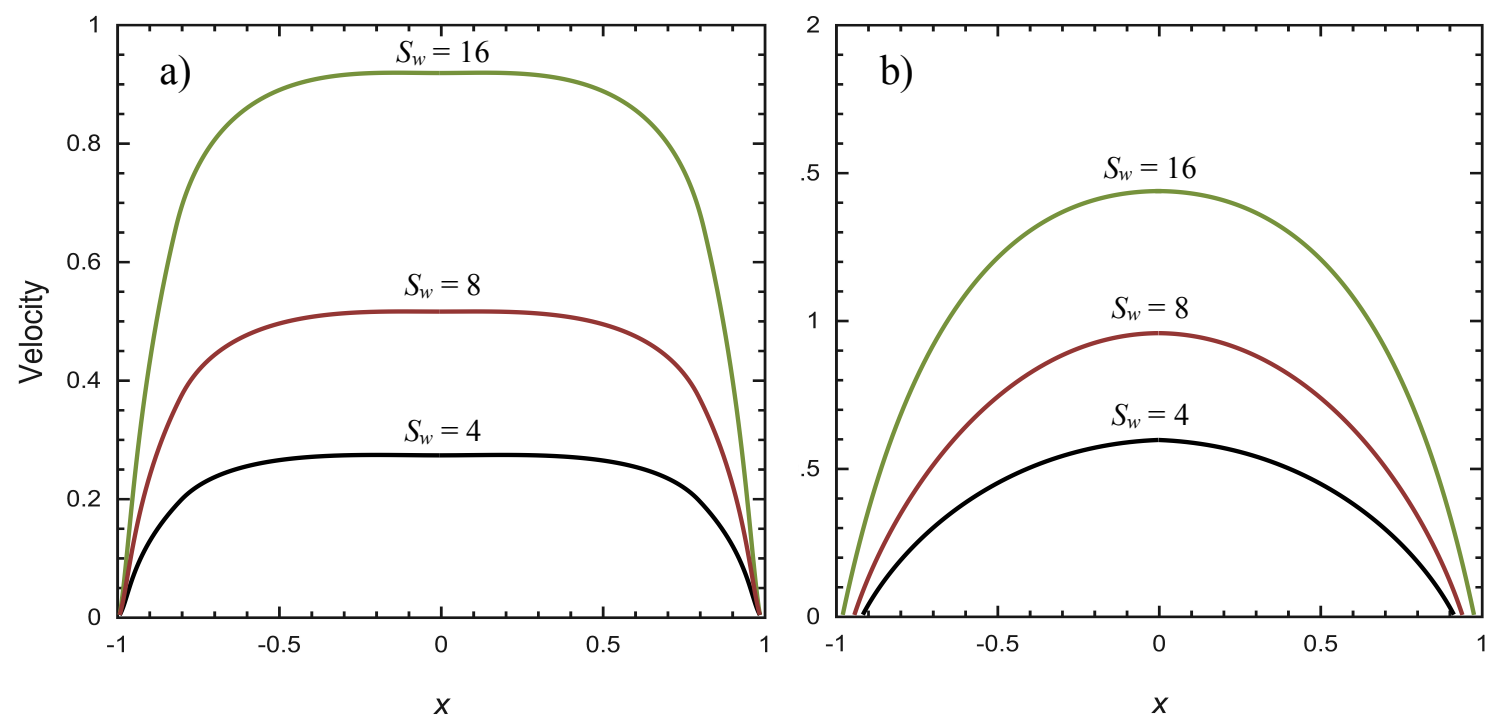

Figure 4. Fluid velocity profile away from the drop for $\mathrm{We}=0.001$, a) $\kappa=8$ and b) $\kappa=2$. 
An interesting axisymmetric vortex flow was observed inside the droplet when it was positively or negatively charged with different internal flow directions for each charge (Figure 5). Similar results were also observed by Huang and Lee (Huang and Lee, 2012) and the explanation for this phenomena is the competition between hydrodynamic dragging forces and the electric forces acting on the surface of the drop. Figure $5 \mathrm{a}$ ) and b) shows the vectors of the fluid velocity relative to the drop velocity for a particular case. Similar vortexes were found in all the cases studied in this research project.

For the negatively charged drop the fluid moves slower than the drop which is evidenced by the downward relative velocity vectors outside the drop on the surrounding fluid. However, the fluid that is immediately adjacent to the drop surfaces on its sides is actually moving faster than the drop. This is explained by the fact that negative charges on the drop surface create an electric field pointing away from the drop. The resulting force due to this electric field acts locally on the adjacent fluid making it move faster. As a result, the fluid inside the drop displays the vortex flow as shown in Figure 5 a).

The exact opposite is observed on the positively charged drop. In the bulk solution, the vectors pointing upwards indicate that the surrounding fluid is moving faster than the drop, which does not occur on the fluid immediately adjacent to the drop surface. The positive charges on the drop surface create an electric field pointing towards the drop. The resulting electrical force in this case acts slowing the adjacent fluid and creates a vortex on the opposite direction to the one observed previously.

a)

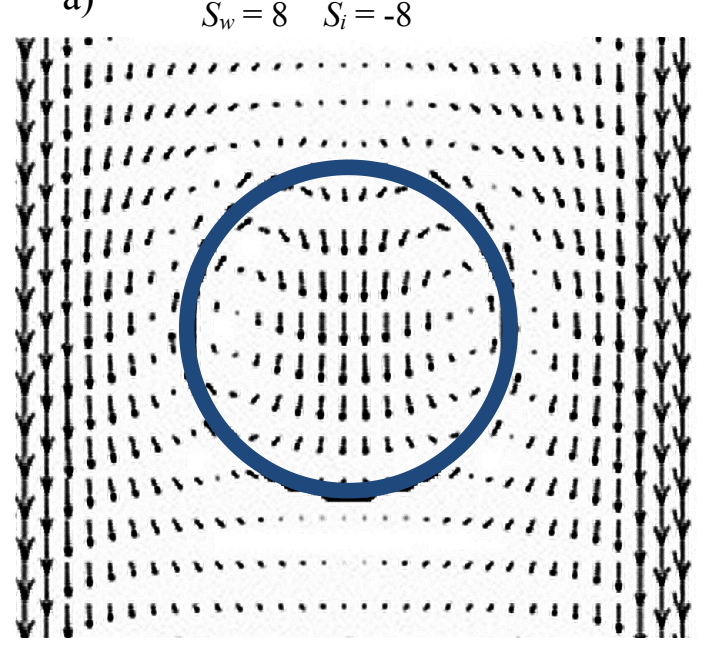

b)

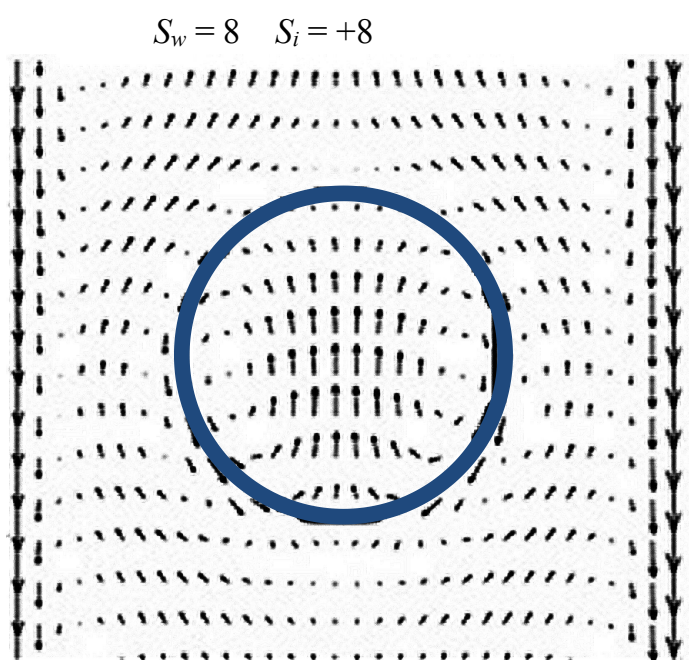

Figure 5. Velocity of the fluid relative to the drop for $\mathrm{We}=0.001, \kappa=8, S_{w}=8$ a) $S_{i}$ $=-8$ b) $S_{i}=+8$.

Such phenomenon is not observed when the drop surface is uncharged as the fluid inside the drop moves at the same velocity of the surrounding fluid. This is due to the absence of 
sufficient surface charges to create electric field normal to the drop surface. The lack of a strong normal electric field and electric forces acting on the drop surface allow the fluid to be carried along with the solution mainly by viscous forces. The observation of these vortexes generated by electro-osmotic flow is particularly relevant for the application of chaotic electro-osmotic flow fluid mixing (Peng and Li, 2015), as they can enhance the mixing rates.

\subsubsection{Charge distribution and Electric field magnitude}

Figure 6 shows the charge distribution in the microchannel for the case where $\mathrm{We}=0.001$ and the drop is perfectly spherical at steady state. Figure $6 a$ ) and b) give EDL thickness of $\kappa$ $=8$ and $\kappa=2$ respectively, fix wall charge density $S_{w}=8$ and drop surface charge density of $S_{i}=-8,0$ and 8 .

As seen from Figure 6 a), an inverse Debye length parameter of $\kappa=8$ gives a thin EDL. Through the major part of the microchannel width the charge concentration in the bulk solution is zero. This is consistent with the plug flow profile of the electro-osmotic flow observed for this case (Figure $4 \mathrm{a}$ ). On the other hand, Figure $6 \mathrm{~b}$ ) evidences that for $\kappa=2$, the thickness of the electric double layer is greater than the radius of the channel and therefore a negative charge accumulation is observed throughout the entire channel. This explains why the plug flow profile was not observed for this case (Figure $4 \mathrm{~b}$ ). Davidson and co-workers (Davidson et al. ,2015) have also shown that in a pressure-driven flow, the choice of $\kappa=2$ allows the EDL to extend to the centreline of the channel and the charge remains negative.

For both $\kappa=8$ and $\kappa=2$, the positively charged drop displayed an accumulation of negative charge around the interface. It can be seen from Figure 6 that the accumulation of negative charges was greater at the top of the drop. This can be explained by looking at the electrolyte solution movement, which is due to bulk convection, the diffusion and ion migration (Davidson et al., 2015). Although the diffusion component should be uniform around the drop due to the constant charge density, the convection component of the fluid movement will tend to keep the ions at the top, or at least slow down the rate that it is distributed to the lower parts of the drop, since the fluid is moving upwards relative to the drop.

Figure 6 also shows that for $\kappa=8$ (thinner EDL), the positive charge accumulation around the negatively charged drop was greater at the top. Similarly, the diffusion component should be uniform around the drop due to the constant charge density, but in this case, the convection component of the fluid movement will tend to drag the ions to the bottom of the drop, as the surrounding fluid is moving downwards relative to the drop. As shown in Figure $6 \mathrm{~b}$ ), the negatively charged drop with $\kappa=2$ did not have a positive charge accumulation surrounding it. Despite the attraction of positive charges to the region near the drop surface, the magnitude of the negative charges in the bulk solution was high enough to prevent a positive charge distribution. However, the effect of the convection component of the movement is still evident from the shape of the 'less negative' region surrounding the drop. Since the drop is moving slower than the surrounding fluid, the effect on charge distribution was greater at its top. 


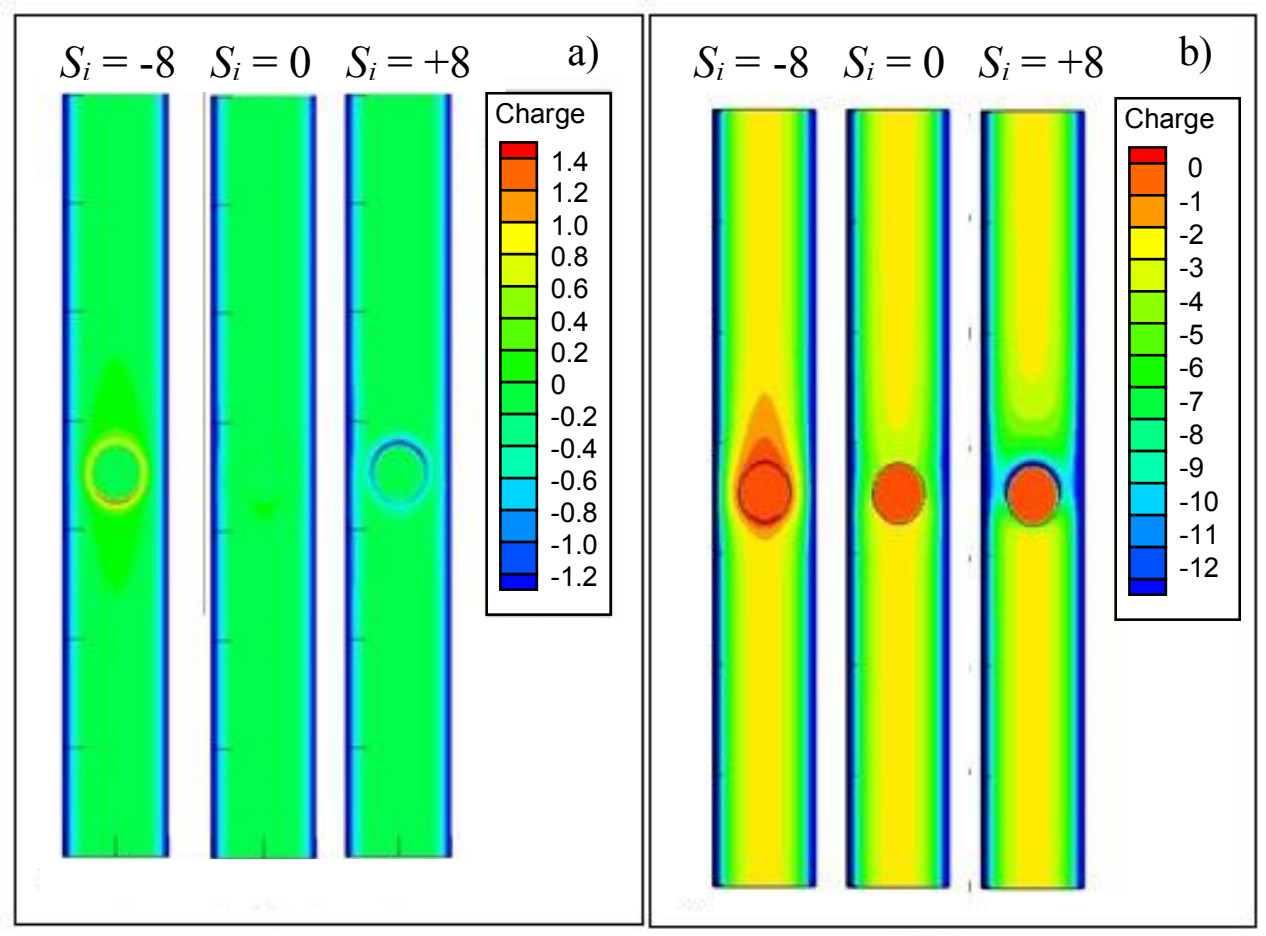

Figure 6. Charge distribution for $\mathrm{We}=0.001$, a) $\kappa=8, S_{w}=8$ and $S_{i}=-8,0$ and +8 b) $\kappa$ $=2, S_{w}=8$ and $S_{i}=-8,0$ and +8 .

The magnitude of the total electric field $\mathrm{E}$ inside the channel is presented in Figure 7 for the case study of $\mathrm{We}=0.001, \kappa=8$ and $\kappa=2$, with wall charge density $S_{w}=8$ and drop interface charge density $S_{i}=-8,0$ and 8 . This field is a combination of the applied electric field, the electric field due to the free charges in the electric double layer near the wall and the drop surface.

For $\kappa=8$ (Figure 7 a), the total electric field is constant in the bulk solution and equal to the applied electric field. Close to the wall, the electric field is greater than 10 due to the overlap of the electric double layers surrounding the drop interface and the wall. It is also observed that there is an enhancement of the total electric field inside the drop, where the fluid has a lower permittivity. This enhancement is also observed on the sides of the drop, which can be explained by the overlapping of electric double layers from both drop and wall charges. Although it may not be clear for the chosen colour scale, the total electric field in the microchannel of $\kappa=2$ (Figure $7 \mathrm{~b}$ ) is slightly higher than 10 , which is caused by the excess of negative charges along the channel.

It can also be seen from Figure 7 that for both $\kappa=8$ and $\kappa=2$ that there is a depletion of the total field on the top of the negatively charged drop and at the bottom of the positively charged drop. In the first case, the negative charges from the surface create a field pointing inwards to the drop. At the bottom, this field is upwards and therefore cancels out the applied field decreasing the overall $E$. The opposite occurs to the positively charged drop, as it 
creates an electric field pointing outwards from the drop and at the top it cancels out the applied field decreasing the overall electric field. Careful analysis of the Figure 7 shows that there is a slight negative charge accumulation near the surface of the uncharged drop due to the advection of ions in the electrolyte solution. This accumulation can explain why there is an enhancement of electric field on the sides and depletion at the top and bottom of the drop.

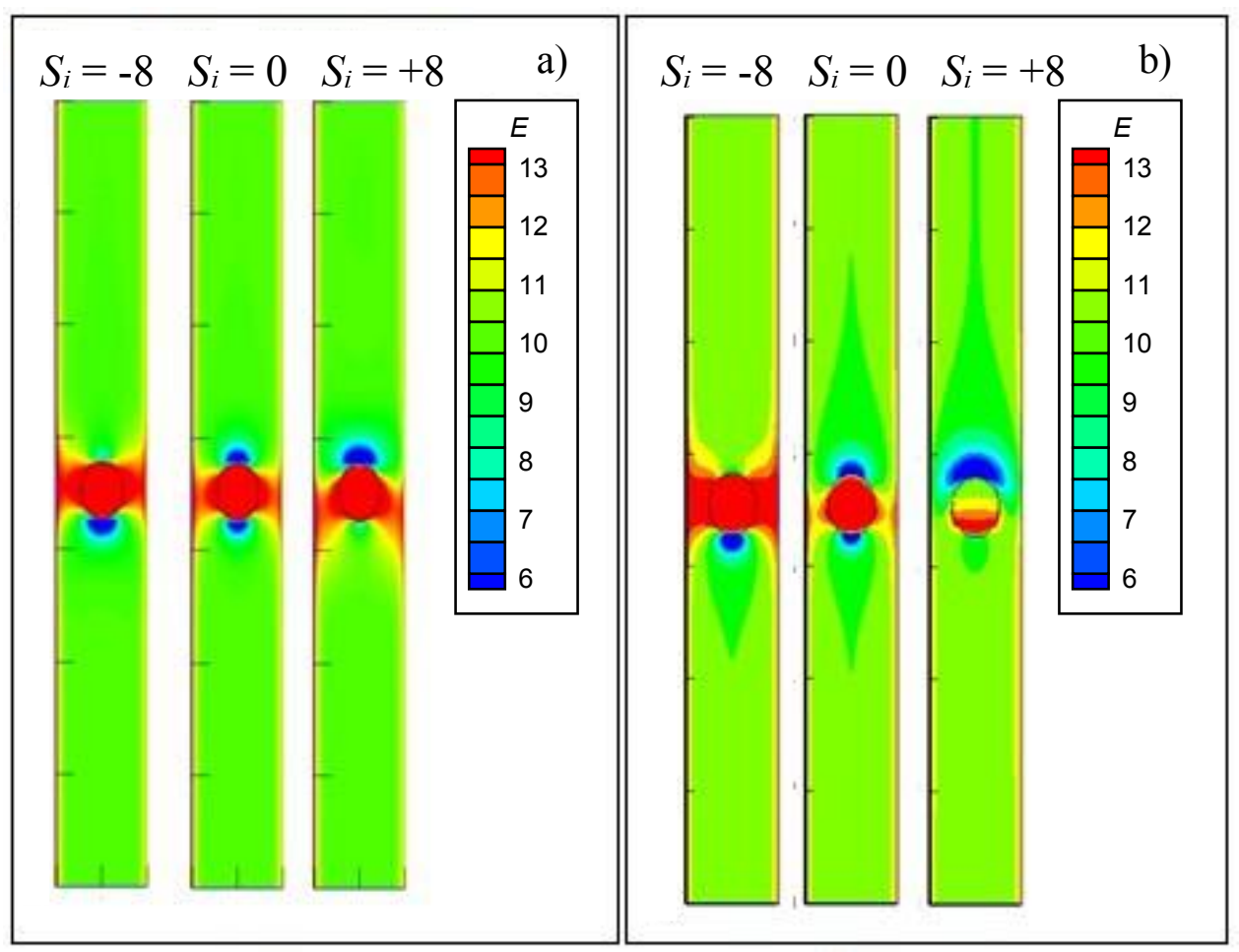

Figure 7. Total Electric Field magnitude for a) $\mathrm{We}=0.001$, a) $\kappa=8, S_{w}=8$ and $S_{i}=-8,0$ and +8 b) $\mathrm{We}=0.001, \kappa=2, S_{w}=8$ and $S_{i}=-8,0$ and +8 .

\subsection{Case study two: Drop with deformation}

\subsubsection{Effect of wall and drop surface charge density on drop velocity}

The results of the drop velocity at the steady state from case study two are shown in Figure 8.

Similar to case study one, the drop velocities were greater for a lower $\kappa$ and increased as the wall charge density increased. The velocities of the uncharged drops were very close to the velocities from case one and the effect of the drop surface charge density was shown to be greater for the case with $\mathrm{We}=0.008$. This means that the negatively charged drops moved even faster and the positively charged drops moved even slower when compared to the drops of same $\kappa$ and wall charge density from case study 1 . The same vortex flows that occurred on case study one was observed for this case. 


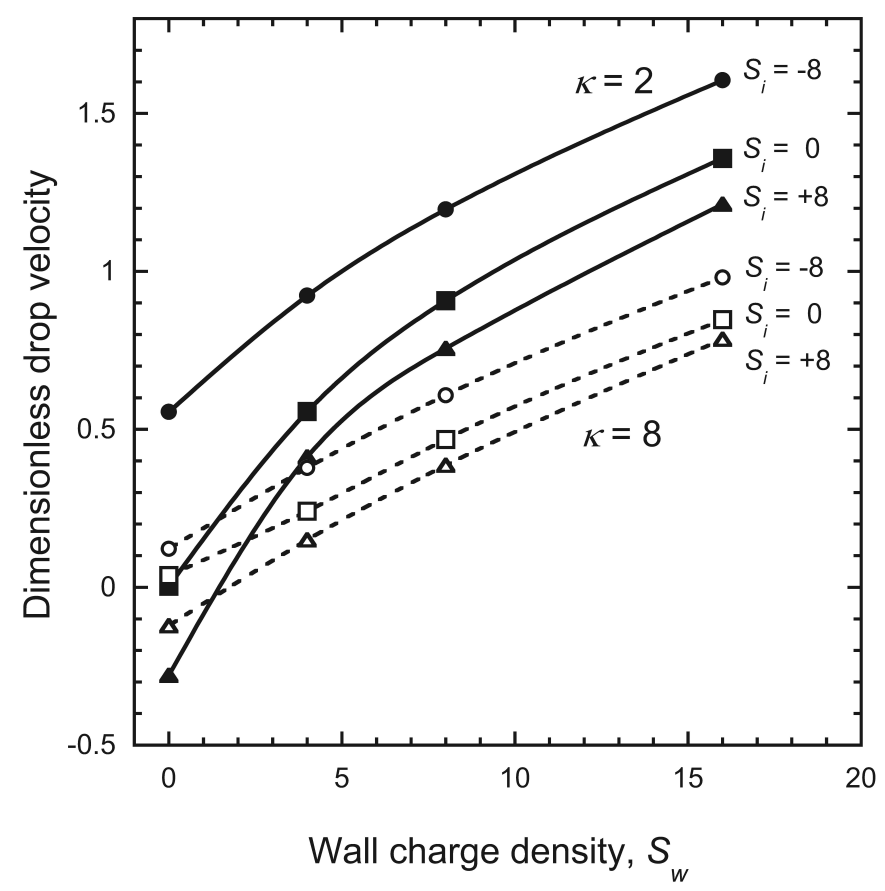

Figure 8. Dimensionless drop velocity at steady state for $\mathrm{We}=0.008$. The lines are guides for the eye. The continuous lines and filled symbols corresponds to $\kappa=2$, and the dashed lines and opened symbols corresponds to $\kappa=8$. In both cases the circles are for $S_{i}=-8$, squares for $S_{i}=0$ and triangles for $S_{i}=+8$.

\subsubsection{Drop deformation}

In thisthis case study, the surface tension was decreased for a Weber number $=0.008$. The reduction of surface tension allowed for electric forces to act more incisively on the surface of the drop and cause some degree of elongation. Drop elongation when in the presence of charged walls was also found for a pressure-driven flow with similar conditions by Davidson and co-workers (Davidson et al. ,2015). It was observed that for higher and lower wall charge density the deformation was very similar to the one in Figure 9 for $S_{w}=8$ (Results not shown). This is a evidence that the effect of increasing the surface charge density on drop deformation is small when compared to changing the ionic concentration of the bulk solution, defined in this research by the Debye length parameter, $\kappa$.

It is evident from Figure 9 that the uncharged drop suffered less deformation than the charged ones. This is explained by the smaller effect of the electrokinetic forces due to charge distribution around the drop. For the deformation of the charged drops, three factors are potentially influencing the deformation. The first factor is the hydrodynamic forces from the fluid adjacent to the drop having a more significant impact on the drop shape, due to the lower surface tension of the drop. The second factor is the charge forces that the counter ions distributed around the charged surface of the drop exerted on it. And the third factor is the permittivity force due to the permittivity jump on the surface, given that the permittivity ratio 
was $\epsilon_{\mathrm{d}} / \epsilon_{\mathrm{c}}=0.025$. These electro kinetic forces are also enhanced by the presence of the applied electric field through the microchannel. A similar inwards lateral force due to permittivity jump in combination with electric field was also found by Davidson and coworkers (Davidson et al. ,2015) for a case with charged walls and charged drop interface.

Comparing the different EDL thickness, it is possible to conclude that for a thinner EDL (Figure $9 \mathrm{a}$ ), $\kappa=8$ ) the deformation of both negatively and positively charged drops were very similar, being influenced mainly by the permittivity jump and the distribution of charges surrounding the drop. For a thicker EDL (Figure $9 \mathrm{~b}$ ), $\kappa=2$ ), on the other hand, the deformation of the negatively charged drop was visibly greater than the positively charged drop. This is explained in more detail in the next section.

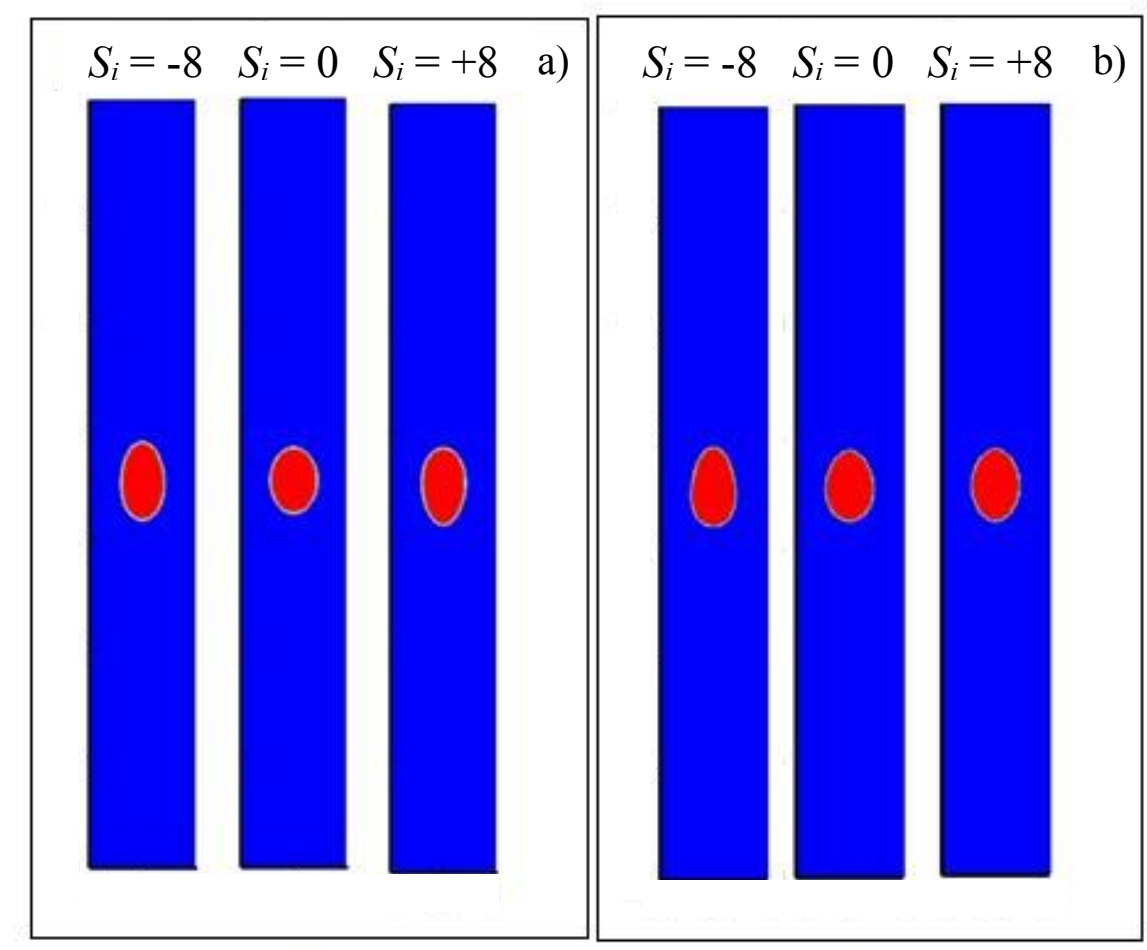

Figure 9. Drop contour for $\mathrm{We}=0.008$, a) $\kappa=2, S_{w}=8$ and $S_{i}=-8,0$ and 8 b) $\kappa=8, S_{w}$ $=8$ and $S_{i}=-8,0$ and 8 .

\subsubsection{Effect of charge distribution and Electric field on drop deformation}

Figure 10 shows the charge distribution in the microchannel for the case where $\mathrm{We}=$ 0.008 and the drop experiences some deformation. Figure 10 a) and b) regard EDL thickness of $\kappa=8$ and $\kappa=2$ respectively, for fix wall charge density $S_{w}=8$ and drop surface charge density of $S_{i}=-8,0$ and 8 . 

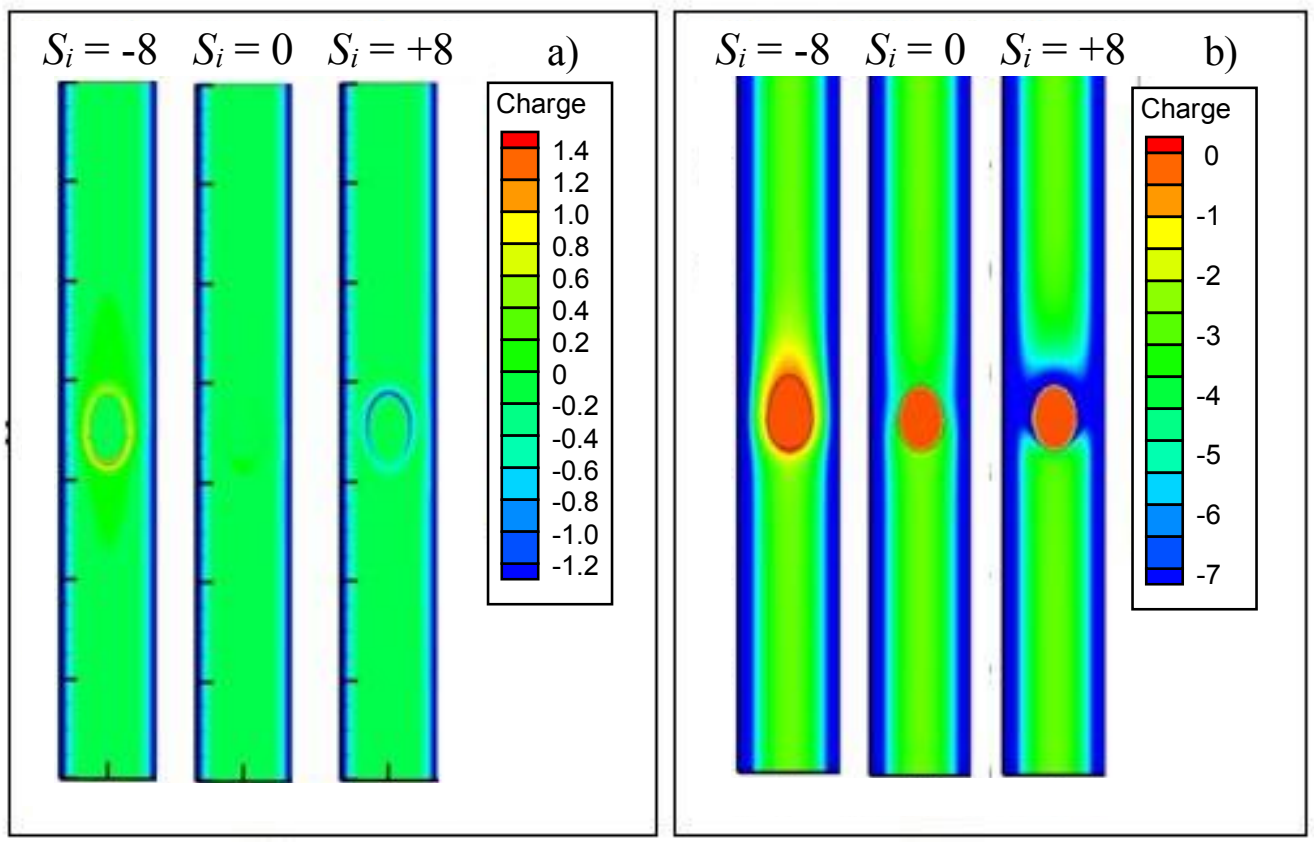

Figure 10. Charge distribution for $\mathrm{We}=0.008$, a) $\kappa=8, S_{w}=8, S_{i}=-8,0$ and +8 b) $\kappa$ $=2, S_{w}=8$ and $S_{i}=-8,0$ and +8

Similar to case study one, the distribution of charges around the drop was shown to be non-uniform due to hydrodynamic and electrokinetic effects. It is clear from Figure 10 a) and b) that the EDL for $\kappa=8$ is very thin when compared to the channel radius and for $\kappa=2$ it extends through the centreline of the channel leaving it somewhat negative. For $\kappa=8$ (Figure 10 a), the distribution of positive charges around the negatively charged drop was higher at the bottom of the drop while the distribution of negative charges around the positively charges drop was higher at the top. The explanation is equivalent for case study one and was fully explained in section 3.1.3. Furthermore, for $\kappa=2$, the negatively charged drop caused a reduction on the highly negative section of the wall EDL and also decreased the intensity of negative charges accumulation around the drop itself. The positively charged drop, on the other hand, caused this section of the EDL to increase, overlapping the EDL due to the drop surface charges.

The distribution of charges in the microchannel due to charged interfaces in combination with the applied electric field contribute to deformation by applying an electric force (charge force) on the surface of the drop. This effect is combined with the effect of a permittivity force generated by a permittivity jump across the interface (permittivity ratio $\varepsilon_{d} / \varepsilon_{c}=0.025$ ) and in opposition to the surface tension. Since the surface tension and permittivity factors are the same for all the cases presented in Figure 10, it is clear that the charge effects vary for different drop surface charge and the inverse Debye length parameter, which is related to the EDL thickness and ion concentration in the bulk solution. 
For $\kappa=8$ (Figure 10a), the deformation is similar for both negatively and positively charged drops and greater than the uncharged drop. Moreover, for $\kappa=2$ (Figure $10 \mathrm{~b}$ ), the charge effects seem to be affecting more the charge deformation. This is somehow expected, since the entire channel contains excess negative charges due to the EDL near the wall. This is possibly the main reason for the uncharged drop being more deformed than when $\kappa=8$. Furthermore, in this last case, the negatively charged drop deformed more than the positively charged drop.

The magnitude of the total electric field $\mathrm{E}$ inside the channel is presented in Figure 11 for the case study of $\mathrm{We}=0.008, \kappa=8$ and $\kappa=2$, with wall charge density $S_{w}=8$ and drop interface charge density $S_{i}=-8,0$ and 8 . The magnitude of the total electric field $E$ inside the channel is presented in Figure 11 for the case study of $\mathrm{We}=0.008, \kappa=8$ and $\kappa=2$, with wall charge density $S_{w}=8$ and drop interface charge density $S_{i}=-8,0$ and 8 .

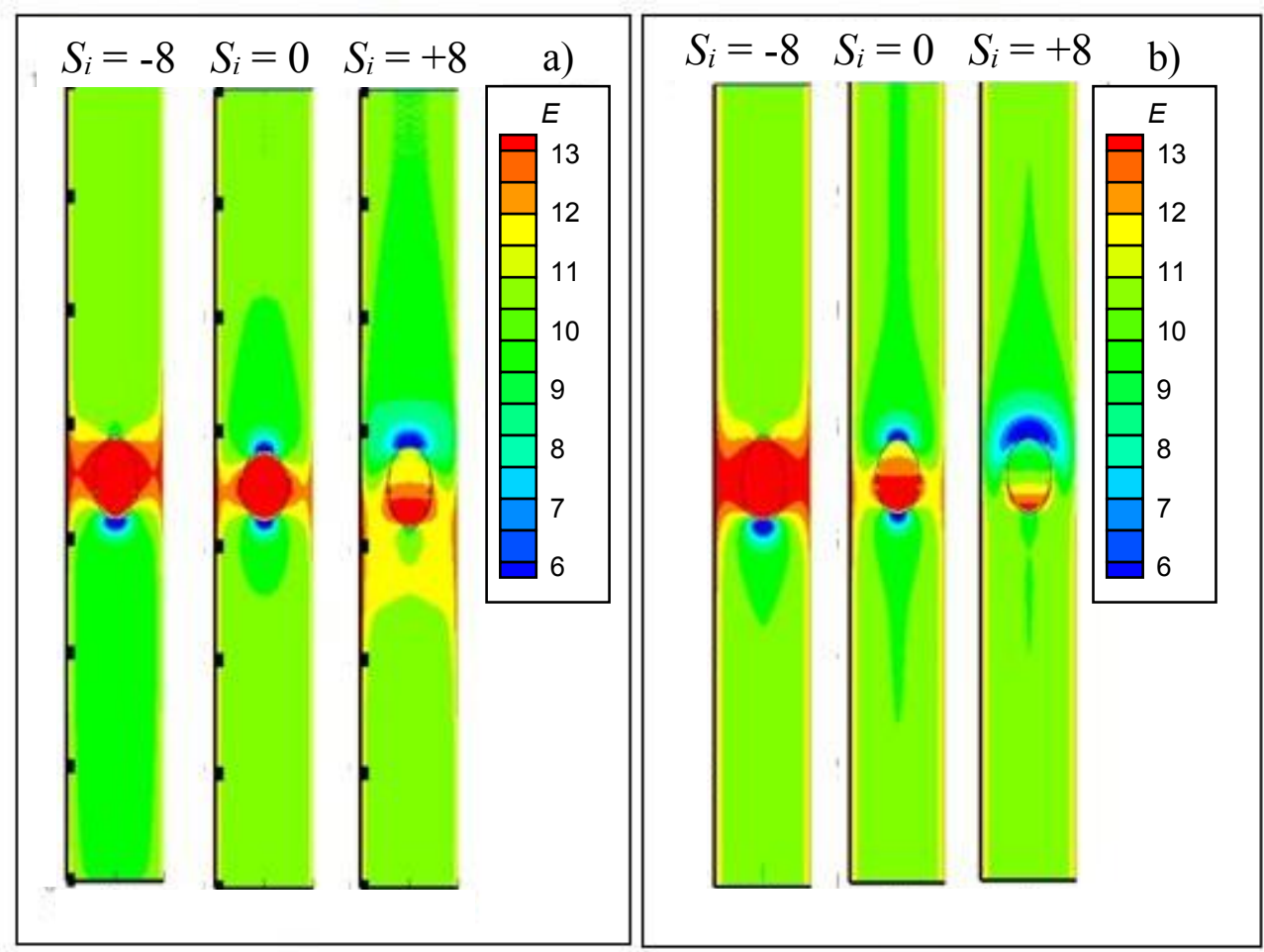

Figure 11. Charge distribution for $\mathrm{We}=0.008$, a) $\kappa=8, S_{w}=8$ and $S_{i}=-8,0$ and +8 b) $\kappa=$ $2, S_{w}=8$ and $S_{i}=-8,0$ and +8

Similar to case study one, the distribution of total electric field is influenced by the application of an external electric field as well as that created by the charge distributions along the channel. In Figure 11, depletion of the total field was observed on the bottom of negatively charged drops, at the top of positively charged drops and on both top and bottom of uncharged drops. This could be caused by the fact that a permittivity jump across the 
interface creates a jump in the electric field in the normal direction to the surface. In addition, the electric field created by the drop surface charges are acting normally to the surface in opposite directions resulting in depletion and enhancement patterns observed.

\subsection{Deformation with no permittivity gradient}

It is known that a combination of charge forces and permittivity forces is responsible for the drop deformation but it is unclear what the individual contribution of each force is to the final result. To further investigate the effect of the permittivity force on drop deformation, some cases were run with no permittivity jump across the drop interface, in other words, both the drop phase and fluid phase had equal permittivity, $\varepsilon_{d}=\varepsilon_{c}=1$. The cases of We $=0.008, \kappa$ $=8, S_{w}=8, S_{i}=-8,0$ and 8 were tested and the results are shown in Figure 12:

a)

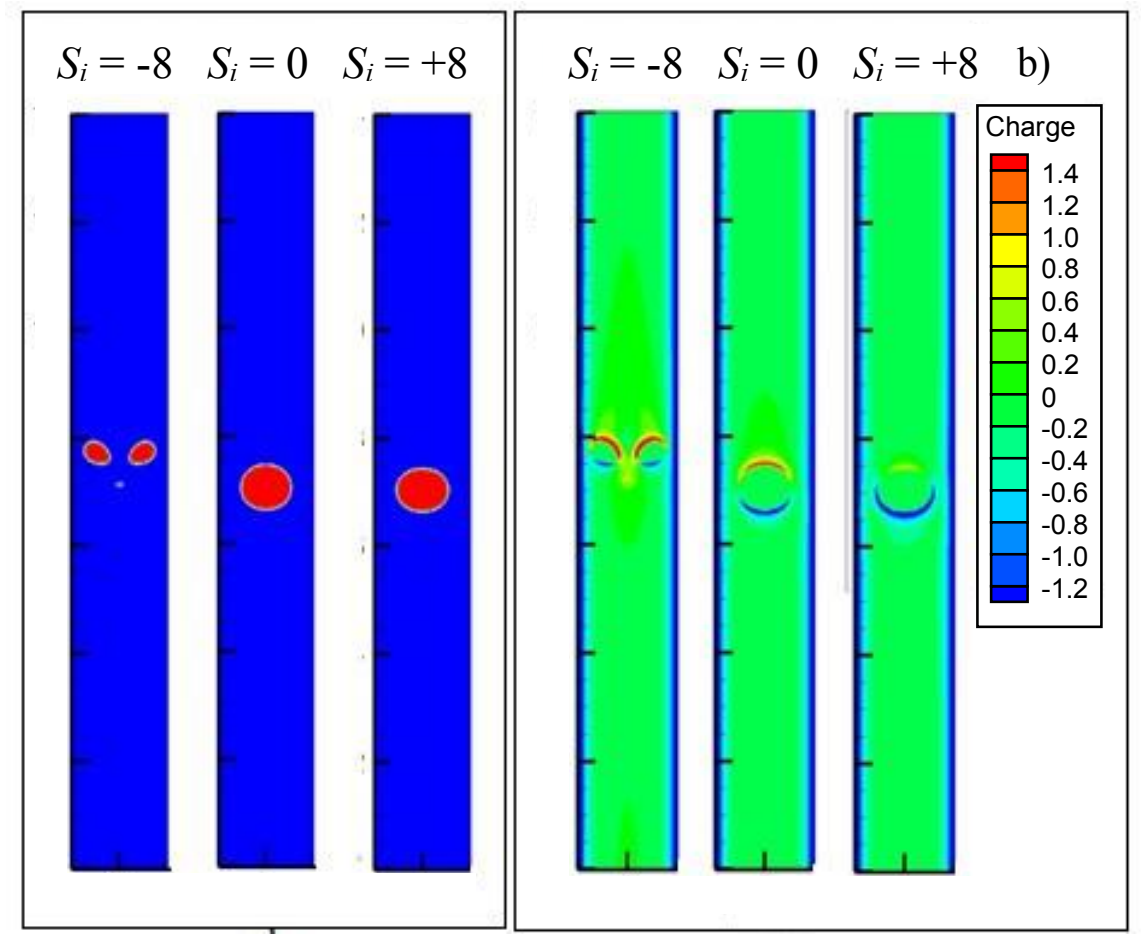

Figure 12. Deformation with no permittivity jump. $\varepsilon_{d}=\varepsilon_{c}=1$, We $=0.008, \kappa=8, S_{w}$ $=8, S_{i}=-8,0$ and 8. a) Drop contour and b) charge distribution.

As seen in Figure 12 a), the drop deformed differently when no permittivity jump was present. Instead of being squeezed from the sides and presenting a vertical elongation like the initial case, a horizontal deformation was observed and in some cases broke into two smaller drops from the central vertical axis. The charge distribution shown in Figure 12 b) suggests that the accumulation of charges at the bottom and top of the drop was even more significant in this case. It is believed that because there were no permittivity forces acting normally to the surface of the drop, the resulting force due to the charge accumulation at the top and 
bottom of the drops was sufficiently strong to cause the drop to flatten. In addition, the hydrodynamic effects of fluid movement being smaller on the surface could also have contributed to the resulting charge distribution. This effect is more evident on the uncharged drop, where the separation of positive and negative charges was very significant at the top and bottom of the drop.

Moreover, the breakup of the negatively charged drop can be explained by a combination of the charge forces and the movement of the drop. The accumulation of positive charges at the top exerted a force into the drop, causing it to flatten up. Since the drop was moving faster than the surrounding fluid and in an opposite direction to the applied force, this force was enhanced and so was the accumulation of positive charges at the top. As the positive charge accumulated, the electric force due to the charge increased even further, resulting in a positive "valley" at the top of the drop causing its breakup. Figure 13 shows the charge distribution at different dimensionless time steps before reaching steady state, supporting the explanation given above.

Initially, there was no charge accumulation and it increased with time. The initial time steps from $t=0$ to $t=40$ have very small charge variation, therefore a charge scale of -1.5 to 2 was used (not shown in Figure 13).

Similar behaviour was not seen in the positively charged drop. This one had an accumulation of negative charges at the bottom. Hence, its deformation was due to charge forces applied from the bottom up. The forces applied on the positively charge drop were on the same direction as the fluid motion and therefore the charge forces were not sufficient to cause drop breakup.

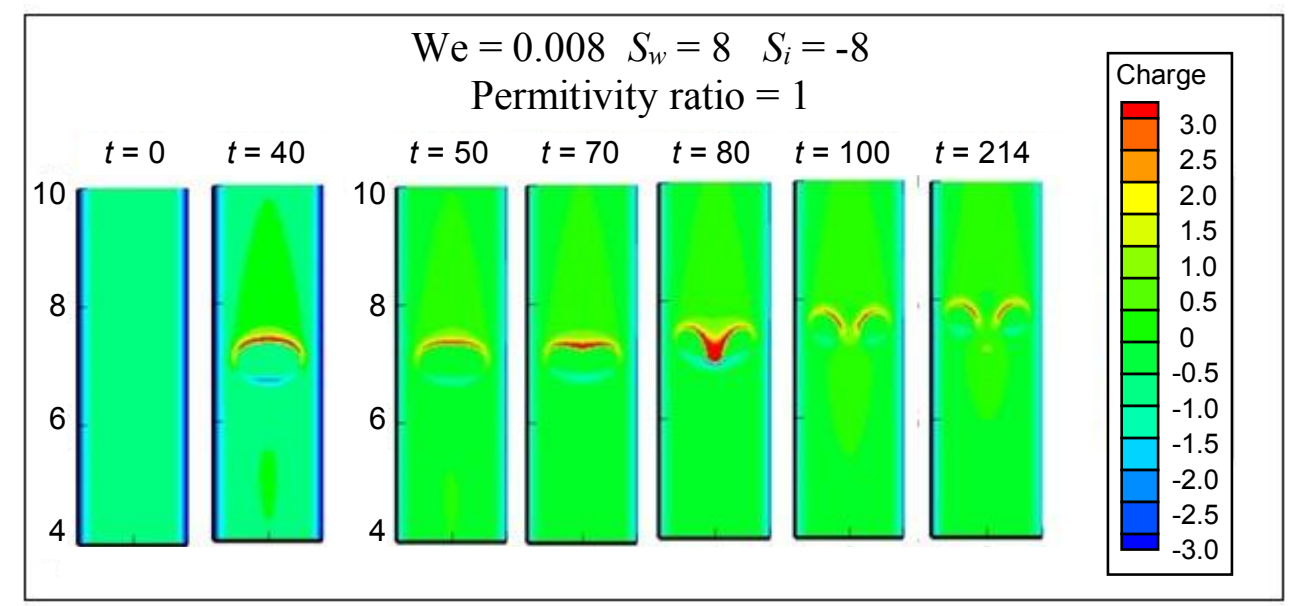

Figure 13. Deformation with no permittivity jump. Charge distribution in dynamic time steps. $\varepsilon_{d}$ $=\varepsilon_{c}=1, \mathrm{We}=0.008, \kappa=8, S_{w}=8$ and $S_{i}=-8,0$ and $+8=8, S_{w}=8, S_{i}=-8$. 


\section{CONCLUSION}

The motion and deformation of a perfectly dielectric drop inside a microchannel filled with an electrolyte solution under electric-driven flow were investigated numerically using a computational dynamic fluid model proposed by Berry and co-workers (Berry et al. ,2013) and extended to account for the presence of charged liquid/liquid interfaces (Davidson et al., 2014). Electrokinetic effects such as charge distribution, electric field magnitude and electric double layer thickness on drop mobility and deformation were analysed. A cylindrical microchannel with fixed dimensionless parameters was used with arbitrary inverse Debye length parameter $\kappa=8$ and $\kappa=2$. The wall was positively charged with increasing surface charge density $\left(S_{w}=0,4,8\right.$ and 16) and the drop surface was either uncharged, positively or negatively charged $\left(S_{i}=0,-8\right.$, or 8$)$. All these cases were divided into two major case studies: the first with $\mathrm{We}=0.001$ giving a spherical drop at steady state and the second with $\mathrm{We}=$ 0.008 which allowed for drop deformation.

In the first case study (We $=0.001$ ), the spherical drop was moving upwards due to electro-osmotic fluid flow caused by the application of the electric field. The drop velocity was generally higher for a lower $\kappa$, which can be explained by a greater area of electroosmotic flow induction due to a thicker EDL. The wall charge density was found to influence the drop and fluid velocities as the velocity increased for higher wall charge density. With regards to the drop charge density, the drop velocity was enhanced when drop surface and wall had negative and positive charges, respectively, and decreased when both had positive charges. This was expected, since the negative charges on the drop surface as well as the negative counter ions of the EDL would tend to move towards the positive electrode while the positive charges on the drop surface would tend to move away from it, hence slowing the drop down.

For $\kappa=8$, the EDL was thin compared to the channel radius which was proven by the plug flow velocity profile inside the channel and the charge distribution being neutral inside. For $\kappa=2$, on the other hand, the EDL was found to be thick enough to extend to the centreline leaving a somewhat negative channel and no plug flow was observed. An interesting axisymmetric vortex flow was observed inside the droplet when it was positively or negatively charged with different internal flow directions for each charge. This phenomenon was due to the competition between hydrodynamic dragging forces and electric forces acting on the surface of the drop.

The charge distribution around the drop was found to be non-uniform due to electrokinetic and hydrodynamic effects on the electrolyte transportation mechanism. There was a greater accumulation of positive charges at the bottom of negatively charged drop and of negative charges at the top of the positively charged drop. This is caused partially by the convection effect of ion transportation and enhanced by the relative velocity of the fluid to the drop being opposite in both cases. The distribution of the total electric field was also nonuniform inside the channel. It was a combination of the applied electric field, the electric field generated by the EDL and the permittivity difference across the drop interface.

In the second case study, the velocity of the uncharged drops remained almost equal to the case study one and the effect of drop charge density in the enhancement or depletion of 
drop mobility was even bigger, with the negatively charged drops moving faster and the positively charged drops moving slower than in case one. Vortex flows inside the drop were also observed. The deformation suffered by the drop was an elongation in the central axis. Charged drops deformed more than uncharged drops, for $\kappa=8$ positively and negatively charged drops deformed equally and for $\kappa=2$ the negatively charged drop deformed more than the positively charged one, which is still to be explained. However, it is known that the deformation is caused by a combination of charge forces as well as permittivity forces acting on the drop surface. Hence, the influence of the permittivity force was investigated by comparing the results with a case with no permittivity jump across the interface. This case presented a horizontal deformation due to the charge accumulations at the top and bottom of the drop. This evidences that the permittivity force was responsible for squeezing the drop from the sides and creating the elongation.

The understanding of drop behaviour and electrokinetic effects in microfluidic systems is extremely important for numerous applications, such as colloid and emulsion industries, micro and nanofluidic, electro-osmotic pumps, and others. This study was an example of the contribution of a new numerical model and how it can be applied to better predict these systems and hence help the design and operation of new technologies.

\section{SUGESTION FOR FURTHER WORK}

The numerical model formulation presented in this study was recently extended to account for liquid-liquid surface charges, which is a significant improvement to the simulations regarding the modelling of droplet motion and deformation in microchannels. Since then, it has been applied to problems involving drop deformation and breakup (Pillai et al., 2015) and compared to analytical solutions for drop relaxation in the presence of ion and interfacial charge and shape evolution of a critically charged water droplet in air (Davidson et al., 2015). To further comprehend the effects of these charged surfaces it would be interesting to simulate previously studied cases, such as the electrophoretic motion of a liquid droplet in a cylindrical pore (Huang and Lee, 2012) to compare previous results and investigate its relevance to this particular study. The effect of fixing the thickness of the electric double layer by fixing the Debye-Hückel parameter on the drop mobility is still unclear and should be given further consideration. A grid refinement to 64 cells resolution showed a variation in drop velocity of approximately $10 \%$, showing that the current resolution of 32 mesh could have limitations on predicting drop behaviour. Hence, a further study with a more refined mesh resolution would be necessary to determine the validity of these results.

The addition of surfactant or emulsifiers to solutions containing droplets can significantly reduce its surface tension (Wallau et al., 2014). Hence, by analysing real systems with large Weber numbers, and therefore low surface tensions, it will be useful to measure the strength of the electrical forces acting on the drop deformation and breakup in nature. This study only considered drops with constant charge density, but depending on the system, the total charge can be fixed and the charge density can vary as the drop deforms and change its surface area. Therefore, using this numerical model with an initial condition set up to such that the total surface charge is constant instead of surface charge density is also a subject of interest. Lastly, a fixed charge density of $S_{i}= \pm 8$ was considered, which is below the critical stability. But, as 
the surface charge increases, the drop can eventually reach a critical Rayleigh instability value and break up due to electrical repulsion forces within the surface charges.

\section{REFERENCES}

BERRY, J. D. D., MALCOLM R.; HARVIE, DALTON J. E. 2013. A multiphase electrokinetic flow model for electrolytes with liquid/liquid interfaces. Journal of Computational Physics, 251, 209-222.

CAMESELLE, C. 2015. Enhancement Of Electro-Osmotic Flow During The Electrokinetic Treatment Of A Contaminated Soil. Electrochimica Acta, 181, 31-38.

DAVIDSON, M. R., BERRY, J. D., PILLAI, R. \& HARVIE, D. J. E. 2015. Numerical simulation of two-fluid flow of electrolyte solution with charged deforming interfaces. Applied Mathematical Modelling.

DAVIDSON, M. R. \& HARVIE, D. J. E. 2007. Electroviscous effects in low Reynolds number liquid flow through a slit-like microfluidic contraction. Chemical Engineering Science, 62, 4229-4240.

DAVIDSON, M. R. B., JOSEPH D.; HARVIE, DALTON J. E. 2014. Numerical simulation of the deformation of charged drops of electrolyte. Advances in Fluid Mechanics X.

GOMES, H. I., RODRÍGUEZ-MAROTO, J. M., RIBEIRO, A. B., PAMUKCU, S. \& DIASFERREIRA, C. 2015. Numerical prediction of diffusion and electric field-induced iron nanoparticle transport. Electrochimica Acta, 181, 5-12.

HUANG, C.-H. \& LEE, E. 2012. Electrophoretic Motion of a Liquid Droplet in a Cylindrical Pore. The Journal of Physical Chemistry C, 116, 15058-15067.

LAC, E. \& SHERWOOD, J. D. 2009. Streaming potential generated by a drop moving along the centreline of a capillary. Journal of Fluid Mechanics, 640, 55-77.

LI, B., ZHOU, W. N., YAN, Y. Y. \& TIAN, C. 2013. Evaluation of electro-osmotic pumping effect on microporous media flow. Applied Thermal Engineering, 60, 449-455.

LI, D. 2004. Electrokinetics in Microfluidics.

LIU, X.-D., FEDKIW, R. P. \& KANG, M. 2000. A Boundary Condition Capturing Method for Poisson's Equation on Irregular Domains. Journal of Computational Physics, $160,151-178$.

PENG, R. \& LI, D. 2015. Effects of ionic concentration gradient on electroosmotic flow mixing in a microchannel. Journal of Colloid and Interface Science, 440, 126-132.

PILLAI, R., BERRY, J. D., HARVIE, D. J. E. \& DAVIDSON, M. R. 2015. Electrolytic drops in an electric field: A numerical study of drop deformation and breakup. Physical Review E, 92, 013007.

RUDMAN, M. 1998. A volume-tracking method for incompressible multifluid flows with large density variations. International Journal for Numerical Methods in Fluids, 28, 357-378.

SAHOO, S., PARAMKUSAM, B. R. \& SRIVASTAVA, R. 2009. Development of a model for electrokinetic study.

SUSARREY-ARCE, A., TIGGELAAR, R. M., MORASSUTTO, M., GEERLINGS, J., SANDERS, R. G. P., GEERDINK, B., SCHLAUTMANN, S., LEFFERTS, L., VAN HOUSELT, A. \& GARDENIERS, J. G. E. 2015. A new ATR-IR microreactor to 
study electric field-driven processes. Sensors and Actuators B: Chemical, 220, 1321.

WALlAU, W., PATEL, R., MUJTABA, I. \& ARELLANO-GARCIA, H. 2014. Electric Field Driven Separation of Oil-water Mixtures: Model Development. In: JIŘÍ JAROMÍR KLEMEŠ, P. S. V. \& PENG YEN, L. (eds.) Computer Aided Chemical Engineering. Elsevier.

WANG, X., WANG, S., GENDHAR, B., CHENG, C., BYUN, C. K., LI, G., ZHAO, M., LIU, S., BYUN, C. K. \& ZHAO, M. 2009. Electroosmotic pumps for microflow analysis. $\operatorname{Tr} A C$ Trends in Analytical Chemistry, 28, 64-74.

\title{
SIMULAÇÃO DE UMA GOTA PERFEITAMENTE DIELÉTRICA NO MOVIMENTO ELETRO-OSMÓTICO DE UM ELETRÓLITO EM UM MICROCANAL
}

\author{
M. M. B. FELISBERTO ${ }^{1}$ e A. V. N. C. TEIXEIRA ${ }^{2}$ \\ ${ }^{1}$ Universidade Federal de Viçosa, Departamento de Química \\ ${ }^{2}$ Universidade Federal de Viçosa, Departamento de Física \\ E-mail: michelle.felisberto@ufv.br
}

\begin{abstract}
RESUMO: Neste trabalho usa-se um modelo de dinâmica de fluidos computacional para simular o movimento e deformação de uma gota dielétrica numa solução eletrolítica em microcanal. Diferentes valores da densidade de carga da parede, dada pelo parâmetro de Debye-Hückel, e do número de Weber foram usados para gotas com interface neutra e carregada positiva ou negativamente. O fluxo e a deformação da gota foram analisados e os efeitos de distribuição de carga, campo elétrico e descontinuidade da permissividade elétrica são discutidos. Em um canal com parede positiva, gotas carregadas negativamente moveram-se mais rápido, enquanto gotas carregadas positivamente moveram-se mais devagar quando comparadas ao movimento de uma gota neutra. Esse efeito aumentou para um valor maior do número de Weber (We). Formações de vórtices foram observadas dentro da gota. Com tensão superficial baixa, as gotas foram alongadas devido à ação de forças elétricas na sua superficie, sendo que as gotas carregadas deformaram-se mais que a neutra. Quando o componente de permissividade da força foi removido, a gota teve uma deformação horizontal que foi suficientemente grande para promover a quebra da gota carregada negativamente.
\end{abstract}

PALAVRAS-CHAVE: Dinâmica de fluidos computacional (CFD); Gota dielétrica; Movimento eletro-osmótico; Solução eletrolítica, Micro canal. 